Article

\title{
A Definite Integral Involving the Logarithmic Function in Terms of the Lerch Function
}

\author{
Robert Reynolds*(D) and Allan Stauffer
}

Department of Mathematics and Statistics, York University, Toronto, ON M3J 1P3, Canada; stauffer@yorku.ca

* Correspondence: milver@my.yorku.ca; Tel.: +1-(416)-319-8383

Received: 20 October 2019; Accepted: 21 November 2019; Published: 24 November 2019

check for updates

Abstract: We present a method using contour integration to evaluate the definite integral of the form $\int_{0}^{\infty} \log ^{k}(a y) R(y) d y$ in terms of special functions, where $R(y)=\frac{y^{m}}{1+\alpha y^{n}}$ and $k, m, a, \alpha$ and $n$ are arbitrary complex numbers. We use this method for evaluation as well as to derive some interesting related material and check entries in tables of integrals.

Keywords: Mellin transform; logarithmic function; definite integral; Hankel contour; Cauchy integral; Bierens de Haan; Prudnikov

\section{Introduction}

The main purpose of this work is to establish a new method which can be used to evaluate the integral $\int_{0}^{\infty} \frac{y^{m} \log ^{k}(a y)}{1+\alpha y^{n}} d y$ in the form of a special function, where $k, \alpha, a, m$ and $n$ are arbitrary complex numbers subject to the restrictions given. This is a novel approach to these problems in mathematics and has been used by us in [1]. This method involves using a form of the Cauchy integral formula. Both the definite integral and special function can be written in terms of the same contour integral, and therefore we can equate the two. This integral and its related special function form a solution space for some well known problems investigated by Prudnikov [2] and Bierens de Haan [3], with other integrals being possible. The integral can be thought of as a Mellin transform, which has been extensively tabulated.

\section{Simultaneous Contour Integrals}

\subsection{Definite Integral of the Contour Integral}

We start with Cauchy's integral Formula (1) where $C$ is the generalized Hankel contour as described by Reynolds and Stauffer [4].

$$
\frac{y^{k}}{k !}=\frac{1}{2 \pi i} \int_{C} \frac{e^{w y}}{w^{k+1}} d w
$$

where the $-1<\operatorname{Re}(w)<0, \operatorname{Im}(w)>0$, and $k$ ! is short for the Gamma function, $\Gamma(k+1)$. We replace $y$ by $\log (a y)$ and multiply both sides by $\frac{y^{m}}{1+\alpha y^{n}}$ in (2) to get the Cauchy equation

$$
\frac{y^{m} \log ^{k}(a y)}{k !\left(1+\alpha y^{n}\right)}=\frac{1}{2 \pi i} \int_{C} \frac{y^{m+w} a^{w}}{\left(1+\alpha y^{n}\right) w^{k+1}} d w
$$


where the definition of the logarithmic function is from Section 4.1 in [5]. This definition of the logarithmic function is used throughout this paper. Then we take the definite integral over $y \in[0, \infty)$ of both sides to get

$$
\begin{aligned}
\frac{1}{k !} \int_{0}^{\infty} \frac{y^{m} \log ^{k}(a y)}{1+\alpha y^{n}} d y & =\frac{1}{2 \pi i} \int_{0}^{\infty} \int_{C} \frac{y^{m+w} a^{w}}{\left(1+\alpha y^{n}\right) w^{k+1}} d w d y \\
& =\frac{1}{2 \pi i} \int_{C}\left(\int_{0}^{\infty} \frac{y^{m+w}}{1+\alpha y^{n}} d y\right) \frac{a^{w} d w}{w^{k+1}} \\
& =\frac{1}{2 i n} \int_{C} \alpha^{-\frac{1+m+w}{n}} a^{w} \csc \left(\frac{\pi(1+m+w)}{n}\right) w^{-k-1} d w,
\end{aligned}
$$

from Equation (3.241.2) in [6]. We replace $y$ with $\alpha^{1 / n} y$ and the integral is valid for $\alpha, a, n$ complex, $-1<\operatorname{Re}(w)<0,-1<\operatorname{Re}(w+m) \leq \operatorname{Re}(n-1)$.

\subsection{Infinite Sum of the Contour Integral}

In this section we will derive an equivalent contour integral by taking an infinite sum over $p$ of a transformed Cauchy integral formula. We once again start with Equation (1), replacing $y$ with $\frac{2 \pi i p}{n}+\frac{\pi i}{n}+\log (a)-\frac{\log (\alpha)}{n}$. Then, we multiply both sides by $-\frac{2 \pi i \alpha-\frac{m+1}{n}}{n} \exp \left(\frac{\pi i(m+1)(2 p+1)}{n}\right)$ to yield

$$
(-2 \pi i) e^{t} \frac{\left((2 p+1) \frac{\pi i}{n}+\log (a)-\frac{\log (\alpha)}{n}\right)^{k}}{\alpha^{(m+1) / n} n k !}=-2 \pi i \int_{C} e^{t} \frac{e^{w\left((2 p+1) \frac{\pi i}{n}+\log (a)-\frac{\log (\alpha)}{n}\right)}}{\alpha^{(m+1) / n} n w^{k+1}} d w .
$$

We then take the infinite sum of both sides of Equation (4) with respect to $p$ over $[0, \infty)$, where $t=\frac{\pi i(m+1)(2 p+1)}{n}$ to get

$$
\begin{aligned}
& -\frac{2 \pi i}{\alpha^{(m+1) / n} n k !} \sum_{p=0}^{\infty} e^{t}\left((2 p+1) \frac{\pi i}{n}+\log (a)-\frac{\log (\alpha)}{n}\right)^{k}=-2 \pi i \sum_{p=0}^{\infty} \int_{C} e^{t} \frac{e^{w\left((2 p+1) \frac{\pi i}{n}+\log (a)-\frac{\log (\alpha)}{n}\right)}}{\alpha^{(m+1) / n} n w^{k+1}} d w \\
& =-2 \pi i \int_{C} \sum_{p=0}^{\infty} e^{t} \frac{e^{w\left((2 p+1) \frac{\pi i}{n}+\log (a)-\frac{\log (\alpha)}{n}\right)}}{\alpha^{(m+1) / n} n w^{k+1}} d w \\
& =\frac{1}{2 i n} \int_{C} \alpha^{-\frac{1+m+w}{n}} a^{w} \csc \left(\frac{\pi(1+m+w)}{n}\right) w^{-k-1} d w,
\end{aligned}
$$

from (1.232.3) in [6], where $\operatorname{csch}(i x)=-i \csc (x)$ from (4.5.10) in [5] and $\operatorname{Im}(w)>0$ for the sum to converge; and if the $\operatorname{Re}(k)<0$, then the argument of the sum over $p$ cannot be zero for some value of $p$. The log terms cannot be combined in general.

\subsection{Equating the Definite Integral and Infinite Sum}

Since the right hand side of Equations (3) and (5) are equal we can equate the left hand sides of these equations to yield

$$
\int_{0}^{\infty} \frac{y^{m} \log ^{k}(a y)}{1+\alpha y^{n}} d y=-\frac{2 \pi i}{\alpha^{(m+1) / n} n} \sum_{p=0}^{\infty} e^{t}\left((2 p+1) \frac{\pi i}{n}+\log (a)-\frac{\log (\alpha)}{n}\right)^{k} .
$$

We can simplify the infinite sum on the right hand side to get

$$
\int_{0}^{\infty} \frac{y^{m} \log ^{k}(a y)}{1+\alpha y^{n}} d y=\frac{e^{\pi i(m+1) / n}\left(\frac{i}{n}\right)^{k-1}(2 \pi)^{k+1} \alpha^{-(m+1) / n}}{n^{2}} \Phi\left(e^{\frac{2 \pi i(m+1)}{n}},-k, \frac{\pi-i n \log (a)+i \log (\alpha)}{2 \pi}\right)
$$

from (9.550) in [6], where $\Phi(r, s, u)$ is the Lerch function, which is a generalization of the Hurwitz zeta function and polylogarithm function, and $n$ is any general complex number. 
The Lerch function has a series representation given by

$$
\Phi(z, s, v)=\sum_{n=0}^{\infty}(v+n)^{-s} z^{n}
$$

where $|z|<1, v \neq 0,-1, \ldots$ and is continued analytically by its integral representation given by

$$
\Phi(z, s, v)=\frac{1}{\Gamma(s)} \int_{0}^{\infty} \frac{t^{s-1} e^{-v t}}{1-z e^{-t}} d t=\frac{1}{\Gamma(s)} \int_{0}^{\infty} \frac{t^{s-1} e^{-(v-1) t}}{e^{t}-z} d t,
$$

where $\operatorname{Re}(v)>0$, or $|z| \leq 1, z \neq 1, \operatorname{Re}(s)>0$, or $z=1, \operatorname{Re}(s)>1$.

Note the left-hand side of Equation (7) converges for all finite $k$. The integral in Equation (7) can be interpreted as a Mellin transform by replacing $m$ by $m-1$ from Equation (17.41) in [6]. The integral in Equation (7) can be used as an alternative method to evaluating the Lerch function. Also note that the right-hand side is defined for all values of $\alpha$ so that if it is real and negative, the integral must be considered as a principle value since there is a singularity in the integrand.

\section{Special Cases of the Logarithmic Integral}

In this section we use Equation (7) with various values of the parameters to yield known integral forms. This illustrates the various forms the integral can take.

3.1. When $k=-1, a=1, n=2 n, \alpha=1$ and $m=m-1$ or $m=q-1$

We get two equations where we look at their difference to yield

$$
\int_{0}^{\infty} \frac{y^{m-1}-y^{q-1}}{\left(1+y^{2 n}\right) \log (y)} d y=-2 \operatorname{arctanh}\left(e^{\frac{i m \pi}{2 n}}\right)+2 \operatorname{arctanh}\left(e^{\frac{i q \pi}{2 n}}\right),
$$

from Equation (1.643.2) in [6] and (4.6.22) in [5], where $0<\operatorname{Re}(m)<\operatorname{Re}(2 n)$ and $0<\operatorname{Re}(q)<\operatorname{Re}(2 n)$. This result is equivalent to equation (4.267.18) in [6] and the case with $n=q$ and some algebraic manipulation forms the solution space for Equations (143.1), (143.2) and (143.5) in [3].

3.2. When $k=-1, a=e^{a i}, \alpha=-1$, and $n=2$

We consider the difference of Equation (7) with $m$ and $-m$. We get, after rationalizing the denominator, transforming the integral in (7) from the domain $y \in[0, \infty)$ to $[0,1]$ and $[1, \infty)$ and replacing $y$ by $1 / y$ in the second integral; then, adding the two integrals over the interval $[0,1]$

$$
\int_{0}^{1} \frac{y^{m}-y^{-m}}{\left(1-y^{2}\right)\left(a^{2}+\log ^{2}(y)\right)} d y=\frac{i^{m} e^{\frac{\pi i(1-m)}{2}} \Phi\left(e^{\pi i(1-m)}, 1, \frac{a}{\pi}\right)-i^{-m} e^{\frac{\pi i(1+m)}{2}} \Phi\left(e^{\pi i(1+m)}, 1, \frac{a}{\pi}\right)}{2 a} .
$$

The term $y^{m}$ in the integral from $[1, \infty)$ contributes to the term $y^{-m}$ in the integral from $[0,1]$ and vice versa, where $-1<\operatorname{Re}(m)<1$ and $\operatorname{Re}(a)>0$, which is Equation (4.282.13) in [6]. The real part of the integral from $[0, \infty)$ containing $\log (y)$ is equal to zero. The numerator is zero at $y=1$ so that the integral exists there in spite of the factor $1-y$ in the denominator.

In the proceeding sections we will use the first derivative with respect to $k$ of Equation (7) and $c=\frac{e^{\pi i(m+1) / n}\left(\frac{i}{n}\right)^{k-1}(2 \pi)^{k+1} \alpha^{-(m+1) / n}}{n^{2}}$ given by

$$
\begin{aligned}
\int_{0}^{\infty} \frac{y^{m} \log ^{k}(a y) \log (\log (a y))}{1+\alpha y^{n} d y} & =c \Phi\left(e^{\frac{2 \pi i(m+1)}{n}},-k, \frac{\pi-i n \log (a)+i \log (\alpha)}{2 \pi}\right) \log \left(\frac{2 \pi i}{n}\right) \\
& -c \Phi^{\prime}\left(e^{\frac{2 \pi i(m+1)}{n}},-k, \frac{\pi-i n \log (a)+i \log (\alpha)}{2 \pi}\right),
\end{aligned}
$$


where $\Phi^{\prime}(z, s, v)$ is the first partial derivative with respect to $s$ of $\Phi(z, s, v)$.

3.3. When $a=1, \alpha=1, n=2$ and $m=0$

We use Equation (12) with $k=0$ to get (13) in terms of $\Phi$ and $\Phi^{\prime}$ on the right-hand side

$$
\begin{aligned}
\int_{0}^{\infty} \frac{\log (\log (y))}{1+y^{2}} d y & =\pi \Phi\left(-1,0, \frac{1}{2}\right) \log (\pi i)-\pi \Phi^{\prime}\left(-1,0, \frac{1}{2}\right) \\
& =\frac{\pi}{4}\left(\pi i+\log \left[\frac{4 \pi^{2} \Gamma^{4}\left(\frac{3}{4}\right)}{\Gamma^{4}\left(\frac{1}{4}\right)}\right]\right),
\end{aligned}
$$

from Equations (12) and (28) in [7]. This result is Equation (1) of Table 148 in [3], where this result is in error.

\subsection{When $a=i, \alpha=1, n=2$ and $m=0$}

we use Equation (12) with $k=0$ to get (14) in terms of $\Phi$ and $\Phi^{\prime}$ on the right-hand side, transform the integral in (12) from the domain $y \in[0, \infty)$ to $[0,1]$ and $[1, \infty)$, and then substitute $1 / y$ for $y$, and add the two over the interval $[0,1]$ to yield

$$
\begin{aligned}
\int_{0}^{1} \frac{\log \left(\frac{\pi^{2}}{4}+\log ^{2}(y)\right)}{y^{2}+1} d y & =\pi \Phi(-1,0,1) \log (\pi i)-\pi \Phi^{\prime}(-1,0,1)-\int_{0}^{1} \frac{\pi i}{y^{2}+1} d y \\
& =\frac{\pi}{2} \log (2)
\end{aligned}
$$

from Equations (12) and (28) in [7]. This result is Equation (13) of Table 147 in [3]. We have also used Equation (7) to check certain integrals tabulated in [2]. These integrals are at once more general than in [2] since all of the parameters in (7) can be complex and the form of the integral contains extra parameters and less general in that the denominator is linear in our work. In particular, we have checked formulas 7, 9, 10, 11, 12 and 15 in Section 2.6.4 as well as 15, 17 and 18 of Section 2.6.5.

\section{Summary}

In this paper we derived a closed form solution of the powers of the logarithm function divided by a generalized denominator in terms of the Lerch function. We then used this integral formula to derive known results for famous integrals. Some of these integrals have been derived using other methods involving a different contour integral. We were able to produce a closed form solution for integrals in Bierens de Haan not previously solved. We also checked various tabulated integrals in Prudnikov in order to verify that they are equivalent to our result. The results in this article were numerically verified for various values, both real and imaginary, using the parameters in the integrals from Mathematica by Wolfram.

Author Contributions: Writing—original draft preparation and conceptualization, R.R.; writing—review and editing, supervision and funding acquisition, A.S.

Funding: This research is supported by NSERC Canada under grant 504070.

Acknowledgments: The authors are grateful to both the referees and the editor for their useful comments and suggestions, which have greatly helped to improve the presentation of this paper.

Conflicts of Interest: The authors declare no conflict of interest.

\section{References}

1. Reynolds, R.; Stauffer, A. Definite Integral of Arctangent and Polylogarithmic Functions Expressed as a Series. Mathematics 2019, 7, 1099. [CrossRef]

2. Prudnikov, A.P.; Brychkov, Y.A.; Marichev, O.I. Integrals and Series, Elementary Functions; USSR Academy of Sciences: Moscow, Russia, 1990; Volume 1. 
3. Bierens de Haan, D. Nouvelles Tables D'intégrales Définies; C.G. van der Post: Amsterdam, The Netherlands, 1867.

4. Reynolds, R.; Stauffer, A. Reciprocal Logarithmic Integrals Expressed as a Series. arXiv 2018, arXiv:1906.04927.

5. Abramowitz, M.; Stegun, I.A. (Eds.) Handbook of Mathematical Functions with Formulas, Graphs, and Mathematical Tables, 9th ed.; Dover: New York, NY, USA, 1982.

6. Gradshteyn, I.S.; Ryzhik, I.M. Tables of Integrals, Series and Products, 6th ed; Academic Press: Cambridge, MA, USA, 2000.

7. Guillera, J.; Sondow, J. Double integrals and infinite products for some classical constants via analytic continuations of Lerch's transcendent. Ramanujan J. 2008, 16, 247. [CrossRef]

(C) 2019 by the authors. Licensee MDPI, Basel, Switzerland. This article is an open access article distributed under the terms and conditions of the Creative Commons Attribution (CC BY) license (http:// creativecommons.org/licenses/by/4.0/). 